\title{
Topological Model of the Pseudogap State: Experimental Signatures
}

\author{
M. C. Diamantini ${ }^{1}$, C. A. Trugenberger ${ }^{2}$, A. T. Bollinger ${ }^{3}$, V. M. Vinokur ${ }^{4,5 *}$ and I. Božović ${ }^{3,6 *}$ \\ ${ }^{1}$ NiPS Laboratory, INFN and Dipartimento di Fisica e Geologia, University of Perugia, Perugia, Italy, ${ }^{2}$ SwissScientific Technologies \\ SA, Geneva, Switzerland, ${ }^{3}$ Brookhaven National Laboratory, Condensed Matter Physics and Materials Science Division, Upton, \\ NY, United States, ${ }^{4}$ Terra Quantum AG, Rorschach, Switzerland, ${ }^{5}$ Physics Department, City College of the City University of New \\ York, New York, NY, United States, ${ }^{6}$ Department of Chemistry, Yale University, New Haven, CT, United States
}

We summarize the basic ideas of our topological model of the pseudogap state of high temperature superconductors (HTS) as a condensate of charged magnetic monopoles, with a focus on new experimental signatures. These include the surface quantum Hall effect, the generation of electric fields when applying magnetic fields by the oblique Meissner effect, and the generation of circular electric fields surrounding electric currents by the oblique Ampère law.

\section{OPEN ACCESS}

Edited by:

Amit Agarwal,

Indian Institute of Technology Kanpur,

India

Reviewed by:

Arko Roy,

University of Trento, Italy

Rafael A. Molina,

Spanish National Research Council

(CSIC), Spain

${ }^{*}$ Correspondence:

V. M. Vinokur

vminokour@gmail.com

1. Božović

bozovic@bnl.gov

Specialty section:

This article was submitted to Condensed Matter Physics,

a section of the journal

Frontiers in Physics

Received: 11 August 2021 Accepted: 29 December 2021 Published: 01 February 2022

Citation:

Diamantini MC, Trugenberger CA, Bollinger AT, Vinokur VM and Božović $I$

(2022) Topological Model of the

Pseudogap State:

Experimental Signatures.

Front. Phys. 9:756760.

doi: 10.3389/fphy.2021.756760
Keywords: pseudogap state, magnetic monopole, monopole condensate, superinsulator, dyon

\section{INTRODUCTION}

Elementary physics courses go a long way to teach us that while single electric charges are abundant in everyday life, magnetic charges appear always in dipoles. It took the genius of Dirac in the 1930s to show that this is not correct. Magnetic monopoles can exist if singularities in the electromagnetic fields are admitted, see [1] for a review. Technically speaking, this is the case if the gauge group is compact, i.e., gauge functions are considered as angular variables [2]. While this may sound odd, it turns out that these singularities are unobservable coordinate singularities if the magnetic charges take only certain quantized values proportional to $\hbar$. In this case, magnetic monopoles are the endpoints of infinitely long, quantized vortices that are unobservable even by the Aharonov-Bohm effect and are called Dirac strings. Quantum electromagnetism with magnetic monopoles is thus perfectly self-consistent.

When electromagnetic forces are embedded in grand unified theories of elementary particle interactions (GUTs), their singularities are resolved within tiny inner cores "containing" very heavy particles [1]. Such fundamental monopoles are so heavy that they could have been produced only in the big bang. After 4 decades of active search, however, they remain elusive.

Lighter monopoles, however, could be realized as excitations in emergent condensed matter systems, where the typical energy scales are 12-13 orders of magnitude smaller than the GUT scale. The Maxwell action (in its non-relativistic form) appears indeed as the electromagnetic response of Mott insulators. For certain Mott insulators with emergent granular structure, however, it is indeed the compact version of the gauge theory which is relevant, and monopoles are thus admitted as bone fide excitations [3]. Let us see how this comes about. Consider a granular ground state made of "bubbles" of Cooper pair condensate, with these bubbles distant enough to suppress tunneling, so that the system is indeed an insulator. Each condensate granule is characterized by the phase $\varphi$ of its superconducting order parameter. As a consequence, quantized vortices can form in the spaces between the bubbles. Note that these are Josephson vortices, with no normal cores: they are characterized only by the non-trivial circulations of the phases, see [4] for a review. 
Nothing prevents these vortices from being open, with a monopole-antimonopole pair at their ends, so that magnetic flux is conserved overall. Two cases can arise. If the vortex has tension, then it is observable, typically short and the monopoles are confined into dipoles. When the tension vanishes these vortices become infinitely long and there is no energy cost in making one go from a monopole to infinity and then back to its antimonopole. Since they are quantized they cannot be detected by the Aharonov-Bohm effect. In other words, for vanishing tension the vortices become Dirac strings and one is left with a neutral gas of magnetic monopoles. Granular insulators of this type, emerging, for example, in the vicinity of a superconductorinsulator transition [5], are thus generically characterized by the presence of magnetic monopoles. If the temperature is also low enough the magnetic monopoles are free to Bose condense [3].

Magnetic monopoles can be incorporated into the electromagnetic realm by modifying the inhomogeneous Maxwell equations to include the corresponding monopole current $j_{m}^{\mu}$,

$$
\begin{gathered}
\partial_{\mu} F^{\mu \nu}=e j_{e}{ }^{\nu}, \\
\partial_{\mu} \widetilde{F}^{\mu \nu}=g j_{m}{ }^{\nu},
\end{gathered}
$$

where $\tilde{F}^{\mu \nu}=(1 / 2) \epsilon^{\mu \nu \alpha \beta} F_{\alpha \beta}$ is the dual field strength tensor, $g=$ $(2 \pi / e)$ is the quantized magnetic charge, and we use natural units $c=1, \hbar=1$ and $\varepsilon_{0}=1$. In the following we will review the role the monopoles may play in the physics of the pseudogap state of high- $T_{c}$ superconductors [6-15] and propose the concrete experimental setup to detect them.

The monopoles that we consider carry both the electric charge $2 e$ and magnetic charge $\pi / e(h / 2 e$ in physical units) and are called dyons. The topological theory of high-temperature superconductivity [16] establishes that the pseudogap state is the state harboring a condensate of dyons and that the charges carrying the electric current in this state are stable symmetryprotected fermionic edge dyons. We propose that edge dyons can be detected by measuring magnetic and electric fields that encircle currents carried by these excitations.

\section{THE MEISSNER EFFECT IN SUPERCONDUCTORS}

A Bose condensate of electric charges is a superconductor [4] carrying the dissipationless currents described by London equations

$$
\begin{array}{r}
\partial_{t} q \mathbf{j}_{e}=\frac{1}{\lambda_{\mathrm{L}}^{2}} \mathbf{E}, \\
\nabla \wedge q \mathbf{j}_{e}=-\frac{1}{\lambda_{\mathrm{L}}^{2}} \mathbf{B},
\end{array}
$$

where $q$ is the charge unit, $q=2 e$ for Cooper pairs, and $\lambda_{\mathrm{L}}$ is the London penetration depth. Together with the Ampère's law,

$$
\nabla \wedge \mathbf{B}=q \mathbf{j}_{e},
$$

they imply that both magnetic fields and currents are screened in the superconductor on the scale $\lambda_{\mathrm{L}}$. At magnetic fields below the lower critical field $H_{\mathrm{c} 1}$, the surface currents generate a magnetic field that exactly counterbalances the applied external magnetic fields so that the external magnetic field is expelled from the superconductor. This Meissner state of a superconductor can be viewed as a phase in which vortices, the magnetic excitations of the system, are confined, the energy to separate a vortexantivortex pair being higher than the thermal energy and the supplied magnetic energy.

\section{THE ELECTRIC MEISSNER EFFECT IN SUPERINSULATORS}

At no surprise, magnetic monopole condensate is a dual mirror of Cooper pair condensate [3]. The corresponding "electric London equations" are

$$
\begin{aligned}
\partial_{t} \frac{2 \pi}{f q} \mathbf{j}_{m} & =\frac{1}{\lambda_{\mathrm{E}}^{2}} \mathbf{B}, \\
\nabla \wedge \frac{2 \pi}{f q} \mathbf{j}_{m} & =\frac{1}{\lambda_{\mathrm{E}}^{2}} \mathbf{E},
\end{aligned}
$$

where $f$ parametrizes the effective strength of the Coulomb interaction in the insulating ground state and $\lambda_{\mathrm{E}}$ denotes the "electric penetration depth." Together with the dual Ampère law

$$
\nabla \wedge \mathbf{E}=-\frac{2 \pi}{f q} \mathbf{j}_{m},
$$

they imply that magnetic currents exist only on the sample surfaces. In this case it is the electric field $\mathbf{E}$ that is expelled from the sample interior by the dissipationless monopole surface currents: this is the "electric Meissner effect". In the Meissner state, whatever the applied external voltage is, the electric field is completely screened, so that there is not a response electric current. This is thus a state with an infinite resistance (even at finite temperatures) and it is correspondingly called a superinsulator [5, 17, 18]. In superinsulators, the excitations are electric vortices. Contrary to the magnetic vortices of superconductors, which must be closed or end at the sample boundaries, the electric flux tubes can have sources. So, even in the Meissner state, there are neutral excitations consisting of chargeanticharge pairs bound by the electric flux tubes generating a linear potential. These are the electric equivalent of pions, with the charges playing the role of quarks [19]. In a superinsulator, the electric charges are confined, but at variance to vortex confinement in superconductors, the charge-confining potential is linear and not logarithmic.

\section{OBLIQUE SUPERINSULATORS AND THE PSEUDOGAP}

The described superinsulator is an exact dual mirror of a superconductor. To understand superinsulators, it is sufficient to substitute $\mathbf{E} \rightarrow \mathbf{B}$ and $\mathbf{B} \rightarrow-\mathbf{E}$ and $2 e \leftrightarrow 2 \pi / 2 e$ in all one knows about superconductivity (a value $f \neq 1$ takes into account an 
effective screening of the electric charge). Things can become more exotic though. Indeed, the usual Maxwell action

$$
S_{\mathrm{M}}=\int d t d^{3} \mathbf{x} \frac{1}{2 f^{2}}\left(\mathbf{E}^{2}-\mathbf{B}^{2}\right)
$$

is not the only quadratic functional in the electric and magnetic fields that arises in three dimensions. The so-called $\theta$-term is an additional possibility $[20,21]$ :

$$
S_{\theta}=\int d t d^{3} \mathbf{x} \frac{\theta q^{2}}{4 \pi^{2}} \mathbf{E} \cdot \mathbf{B}=\int d t d^{3} \mathbf{x} \frac{\theta q^{2}}{32 \pi^{2}} F_{\mu \nu} \epsilon^{\mu \nu \alpha \beta} F_{\alpha \beta} .
$$

This is a topological term, which simply means that, due to the presence of the totally antisymmetric tensor $\epsilon^{\mu \nu \alpha \beta}$, it can be formulated independently of a metric, and hence of the geometric details of the manifold. When it appears in the effective field theory for an insulator, its effects are totally transparent to disorder and localization effects, they are determined only by the topology of the sample. When the $\theta$ term is added to the effective action of insulators one gets the famed (strong) topological insulators (for a review see [22]). It turns out that when the gauge symmetry is compact, as explained above, the parameter $\theta$ is an angle: the correct periodicity is $\theta \rightarrow \theta$ $+2 \pi$ for fermionic systems and $\theta \rightarrow \theta+4 \pi$ for bosonic systems [23]. Correspondingly, fermionic topological insulators are characterized by $\theta=\pi$, while bosonic ones have $\theta=2 \pi$.

The topological character of the $\theta$-term can also be established from it being a total derivative

$$
\frac{1}{4} F_{\mu \nu} \epsilon^{\mu \nu \alpha \beta} F_{\alpha \beta}=\partial_{\mu}\left(A_{\mu} \epsilon^{\mu \nu \alpha \beta} \partial_{\alpha} A_{\beta}\right)
$$

Therefore, all the effects of the $\theta$-term are seen at the boundaries of the sample and are governed there by the effective Chern-Simons action [24].

$$
S_{\text {boundary }}=\frac{1}{4 \pi} \frac{\theta q^{2}}{2 \pi} A_{\nu} \epsilon^{\mu \nu \alpha} \partial_{\nu} A_{\alpha} .
$$

What if the topological insulator admits magnetic monopoles? In this case something very peculiar happens: due to the $\theta$-term the magnetic monopoles acquire also an electric charge $\theta / 2 \pi$. This phenomenon is known as Witten effect [25]. The monopoles carrying also the electric charge are called dyons. In [16] we have proposed a topological effective theory of the HTS in which the mysterious pseudogap state is established to be a Bose condensate of $\theta=2 \pi$ dyons, while the superconducting dome harbors a phase-separated coexistence regime of the dyon condensate with the normal Cooper pair condensate, and $\pi / f^{2}$ is identified with a monotonic function of the relative doping ratio $p / p_{\max }$, with $p$ being the doping and $p_{\max }$ being its value at the maximum $T_{c}$. The $\theta$-term, in this case, is responsible for the magneto-electric effect, indeed observed experimentally in the pseudogap state [16]. Let us, therefore, focus on the properties of a dyon condensate.

When dyons Bose condense, we expect a peculiar state to arise "mixing" superconductor and superinsulator features. Indeed, in this case the London equations assume the form

$$
\begin{aligned}
\partial_{t} \frac{2 \pi}{f q} \mathbf{j}_{\mathrm{m}} & =\frac{\Lambda^{2}}{(f q)^{2}}\left(\mathbf{B}+\frac{(f q)^{2}}{2 \pi} \frac{\theta}{2 \pi} \mathbf{E}\right), \\
\nabla \wedge \frac{2 \pi}{f q} \mathbf{j}_{\mathrm{m}} & =\frac{\Lambda^{2}}{(f q)^{2}}\left(\mathbf{E}-\frac{(f q)^{2}}{2 \pi} \frac{\theta}{2 \pi} \mathbf{B}\right),
\end{aligned}
$$

where $\Lambda$ denotes the ultraviolet cutoff of the effective theory. Let us now combine the second of these equations with the (static) Ampère equations

$$
\begin{gathered}
\nabla \wedge \mathbf{B}=f q \frac{\theta}{2 \pi} \mathbf{j}_{\mathrm{m}}, \\
\nabla \wedge \mathbf{E}=-\frac{2 \pi}{f q} \mathbf{j}_{\mathrm{m}},
\end{gathered}
$$

where we have used the fact that the electric current is $\mathbf{j}_{\mathrm{e}}=(\theta / 2 \pi)$ $\mathbf{j}_{\mathrm{m}}$ since it is carried by charged monopoles. Taking the curl of the second equation in (Eq. 10) and using (Eq. 11) we get (for sourceless currents)

$$
\left(\nabla^{2}-\frac{1}{\lambda_{\theta}^{2}}\right) \mathbf{j}_{\mathrm{m}}=0
$$

where

$$
\lambda_{\theta}=\frac{2 \pi}{f q \Lambda} \frac{1}{\sqrt{\left(\frac{2 \pi}{q f}\right)^{2}+\left(\frac{\theta}{2 \pi}\right)^{2}}} .
$$

This shows that all currents are screened in the interior of the dyon condensate and survive only in a surface strip of width $\lambda_{\theta}$. But what happens to the electric and magnetic fields? The London Equation 10 clearly show that now the appropriate axes in the "flavour" electric-magnetic space are tilted. This is the reason why such a state is called an oblique superinsulator [16]. A combination of electric and magnetic fields such that

$$
\mathbf{E}=\frac{(f q)^{2}}{2 \pi \hbar c \varepsilon_{0}} \frac{\theta}{2 \pi} c \mathbf{B}=8 \pi \alpha \frac{f^{2}}{\pi} \frac{\theta}{2 \pi} c \mathbf{B},
$$

(here we have reinstated physical units and have chosen $q=2 e$; $\alpha=e^{2} /\left(4 \pi \varepsilon_{0} \hbar c\right) \approx 1 / 137$ is the fine structure constant) is not screened and can penetrate the oblique superinsulator, without generating surface currents. This combination plays the same role as the electric field in a superconductor. Let us decompose any combination of electromagnetic fields in the orthogonal basis

$$
\left(\begin{array}{c}
\frac{(f q)^{2}}{2 \pi \hbar c \varepsilon_{0}} \frac{\theta}{2 \pi} \\
1
\end{array}\right), \quad\left(\begin{array}{c}
-1 \\
\frac{(f q)^{2}}{2 \pi \hbar c \varepsilon_{0}} \frac{\theta}{2 \pi}
\end{array}\right)
$$

where the upper component is the electric one and the lower component is the magnetic one, with magnetic fields multiplied by $c$ so that they have the same units as electric fields. Combinations along the first basis vector can penetrate the oblique superinsulator, combinations along the second basis vector are expelled; they play the same role as the magnetic fields in superconductors. Let us assume that we apply the external magnetic field $c \mathbf{B}$. We can decompose the corresponding "flavour" vector $(0, c B)$ into its components along the above orthogonal basis and the second component 
will be expelled. Therefore, the oblique superinsulator reacts to the applied field by generating, via surface currents, compensating magnetic and electric fields:

$$
\begin{gathered}
c \mathbf{B}_{\text {comp }}=-\frac{\left(\frac{(f q)^{2}}{2 \pi \hbar c \varepsilon_{0}} \frac{\theta}{2 \pi}\right)^{2}}{1+\left(\frac{(f q)^{2}}{2 \pi \hbar c \varepsilon_{0}} \frac{\theta}{2 \pi}\right)^{2}} c \mathbf{B}, \\
\mathbf{E}_{\text {comp }}=\frac{\frac{(f q)^{2}}{2 \pi \hbar c \varepsilon_{0}} \frac{\theta}{2 \pi}}{1+\left(\frac{(f q)^{2}}{2 \pi \hbar c \varepsilon_{0}} \frac{\theta}{2 \pi}\right)^{2}} c \mathbf{B} .
\end{gathered}
$$

We call this effect the oblique Meissner effect and it constitutes our first experimental prediction: the external magnetic field applied to the HTS material in the deeply underdoped $(f \gg 1)$ pseudogap state induces the electric field

$$
\mathbf{E}=\frac{\pi}{f^{2}} \frac{1}{8 \pi \alpha} c \mathbf{B} \approx 5.45 \frac{\pi}{f^{2}} c \mathbf{B} .
$$

Note that, in the deeply underdoped regime $f \gg 1$, the oblique penetration depth (Eq. 13) becomes very small, so that this topological magnetoelectric effect should be observable for reasonable values of the doping.

In the interior of superconductors, the charges are Bosecondensed, while the "orthogonal" excitations, vortices, are in an out-of-condensate state and confined (in the Meissner state). In the interior of an oblique superinsulator, excitations with (electric, magnetic) charges $(\theta / 2 \pi, 1)$ are in the Bose condensate, while the orthogonal excitations $(1,-\theta / 2 \pi)$ are confined. As in normal superinsulators there are possible neutral dyon-antidyon excitations connected by a string carrying both electric and magnetic flux, oblique pions. Currents are limited to a strip of the width $\lambda_{\theta}$ around the surfaces. Since in this strip region both electric and magnetic fields penetrate the system and are not forced into confining flux tubes, one expects single dyon excitations to arise. In the underdoped regime $f \gg 1$, the surface strips become very thin, so that these dyons behave essentially as $2 \mathrm{D}$ particles, realizing symmetry-protected edge states like those of the quantum Hall effect or of topological insulators [26, 27]. As we have derived previously, the electromagnetic interaction in these quasi-2D strips contains the Chern-Simons term due to the bulk $\theta$-term. This Chern-Simons term converts the surface dyons into fermions via the anyon mechanism. For $\theta=2 \pi$, these symmetry-protected surface fermions with the charge $2 e$ are the only charged low-lying excitations in the pseudogap state. As any fermion living on a $2 \mathrm{D}$ surface, they give rise to the integer quantum Hall effect with Hall conductivity $\sigma_{\mathrm{H}}=(2 e)^{2} / 2 \pi$, as appropriate to fermions with charge $2 e$.

The surface fermionic dyons typically live on a ChalkerCoddington percolation network [28] and, being symmetryprotected, behave on it as a perfect Fermi liquid, giving rise to the resistance proportional to $T^{2}$, as has been indeed observed in the pseudogap state $[29,30]$. Moreover, since dyons carry both electric and magnetic charges, they spontaneously break the purely electric $C_{4}$ symmetry of the antiferromagnetic background lattice to the diagonal $C_{2}$ combined electricmagnetic symmetry, giving rise to the observed nematic effects [31, 32]. And, as we have already mentioned, the observed magnetoelectric Kerr effect [33] follows straight from the very presence of the $\theta$-term, as in topological insulators.

The presence of the fermionic dyons gives rise to more measurable effects. Suppose we produce an electric current $I_{e}$ of these excitations in the pseudogap state and that we encircle the sample by an orthogonal conducting ring. Since each particle in the current carries also a unit of magnetic charge, the dual Ampère law

$$
\mathbf{E}=-\frac{1}{2 \pi r} \frac{1}{\varepsilon_{0}} \mathbf{I}_{m},
$$

second equation in (Eq. 11), implies that a voltage

$$
V=\sqrt{\frac{\pi}{f^{2}}} \frac{1}{\sqrt{\pi} 8 \alpha} \frac{1}{c \varepsilon_{0}} I_{\mathrm{e}} \approx 9.66 \sqrt{\frac{\pi}{f^{2}}} \frac{1}{c \varepsilon_{0}} I_{\mathrm{e}},
$$

is induced in the conducting ring.

\section{A "SMOKING-GUN" EXPERIMENT}

Now we focus on one sharp and unique prediction of the theory presented above, and describe in full detail an experiment that can put this prediction to a rigorous test.

The idea is illustrated in Figure 1. A dc current of electrically charged particles (electron monopoles) running through a straight metallic wire generates a circumferential magnetic field that can be detected and measured, e.g., by deflecting a compass needle (Figure 1A). A dual of this experiment can be performed by running a dc current of magnetically charged particles (magnetic monopoles) through a straight wire of an appropriate material, thus generating a circumferential electric field. The later can be detected e.g., by wrapping around that straight wire a pick-up coil and measuring the electric current in the coil, or if the coil is not completely closed, measuring a dc voltage between the two ends (Figure 1B). The same experiment would work if the low-energy excitations in the material under study were dyons (Figure 1C), with the added advantage that in this case the dc current, simultaneously both electric and magnetic, through the straight central wire can be simply sustained by using a standard current source.

The above illustration is conceptual. To make it practical, and allowing for the possibility that the induced electric field may be very weak, we want to make the coil as close to the central wire as possible. One way to do this is using two techniques, thin film deposition and lithography, that have been developed to a high level for the needs of the semiconductor electronics industry. Our choice of the materials are high-temperature superconducting (HTS) cuprates, since these have been proposed to host dyons in a large part of the temperature-doping phase diagram. Fortunately, in the last couple decades, atomic-layer-by-layer molecular beam epitaxy (ALL-MBE) has been developed to the level of producing atomically perfect single-crystal films of cuprates, down to one 


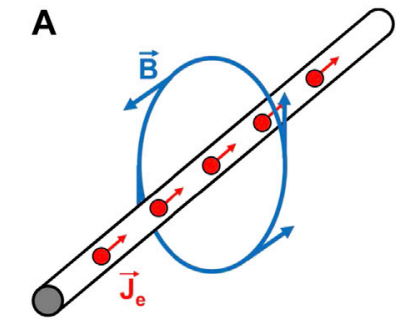

$0=q_{e}$

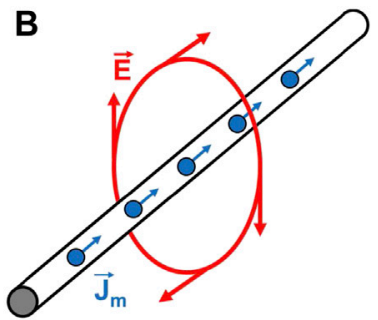

$O=q_{m}$

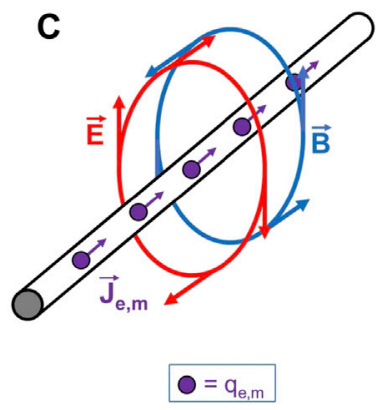

FIGURE 1 | Electrons, magnetic monopoles, and dyons. (A) An electric current induces circumferential magnetic field. (B), A magnetic monopole current induces circumferential electric field, which would therefore be a "smoking gun" experimental signature of the existence of magnetic monopoles. (C), A dyon current induces both circumferential electric and magnetic fields.
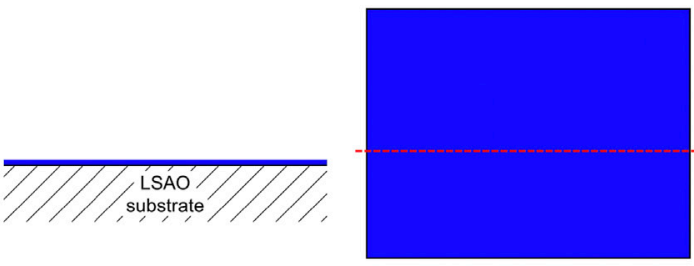

Step 1. Deposit $10 \mathrm{ML}$ of optimally doped LSCO for the first (bottom) superconducting layer.
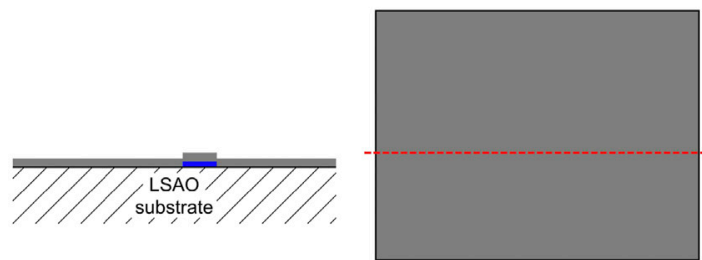

Step 3. Deposit $16 \mathrm{ML}$ of insulator LSAO for the first insulating layer.
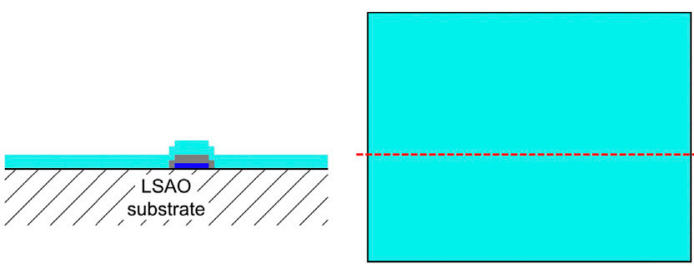

Step 5. Deposit 25 ML for the 'active' LSCO layer.

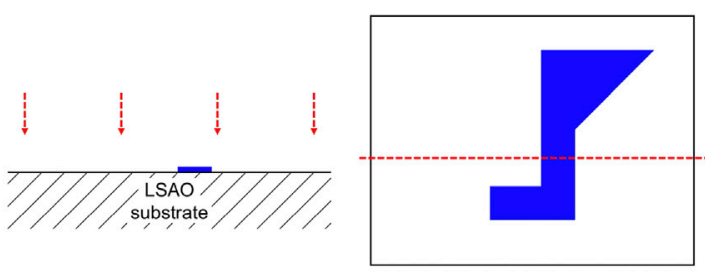

Step 2. Ion-mill part of the bottom superconducting LSCO layer, all the way to the substrate.

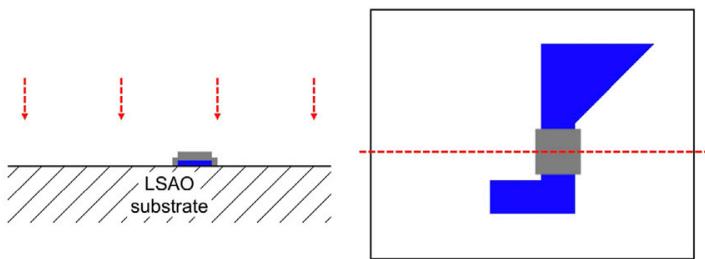

Step 4. Ion-mill the first insulating LSAO layer; leave the bottom SC contact areas intact.

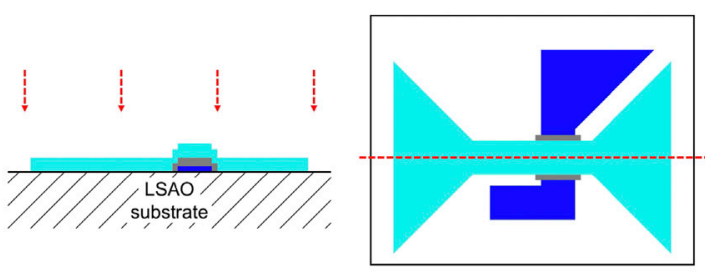

Step 6. Ion-mill the active LSCO layer; leave the bottom SC contact areas intact.

FIGURE 2 |Dyon detection device assembling, stage 1. Left panels: side view; right panels: top view. Dashed line in right panels indicates location of section shown in left panels. 

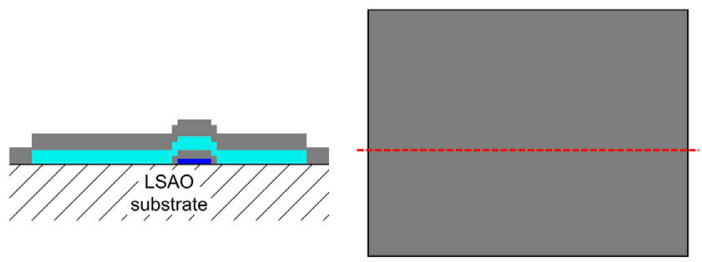

Step 7. Deposit $30 \mathrm{ML}$ of insulator LSAO for the second insulating layer.
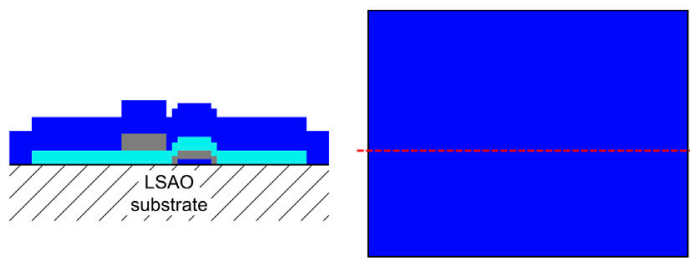

Step 9. Deposit $60 \mathrm{ML}$ of optimally doped LSCO for the second (top) superconducting layer.
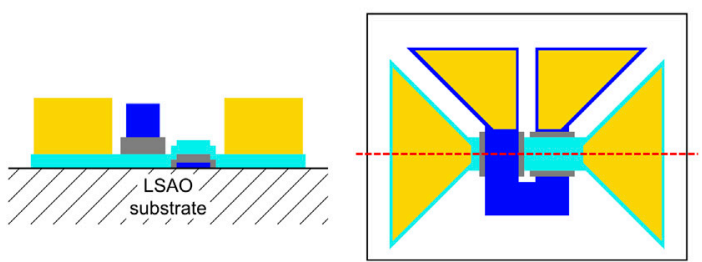

Step 11. Deposit thick gold contact pads on the four contact areas.
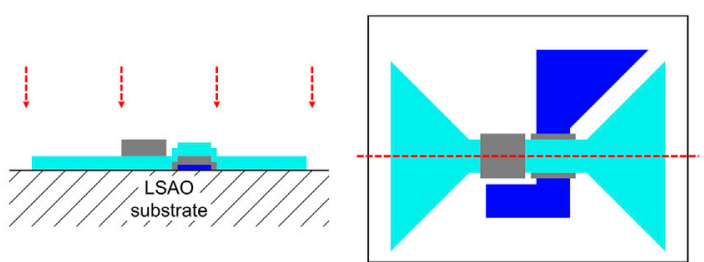

Step 8. Ion-mill the second insulating LSAO layer; leave contact areas intact.

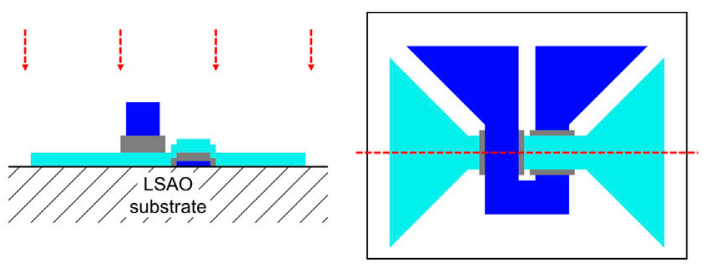

Step 10. lon-mill the second (top) superconducting LSCO layer; leave all four contact areas intact.
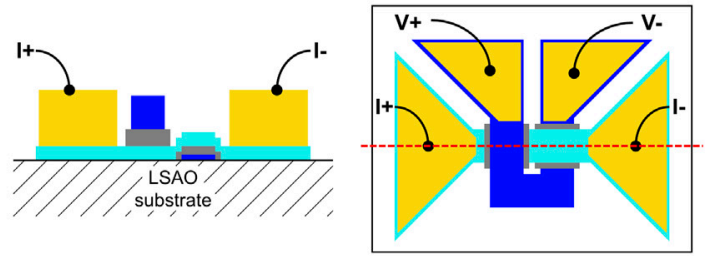

Step 12. Wire-bond to the four LSCO/gold contact pads.

FIGURE 3|Dyon detection device assembling, stage 2. Left panels: side view; right panels: top view. Dashed line in right panels indicates location of section shown in left panels.

unit cell (UC) thick while maintaining the critical temperature $\left(T_{\mathrm{c}}\right)$ as high as that in the bulk crystals [34-36]. Moreover, heterostructures and superlattices have been fabricated with atomically smooth surfaces and interfaces, and engineered down to a single atomic layer. In parallel, we have also developed the lithographic techniques to fabricate HTS devices down to nanometer scale [37]. In what follows, we leverage on these advanced synthetic and lithographic capabilities.

In Figure 2, Figure 3 we show a design of a multilayer thin-film device, envisioned to enable detection of magnetic monopoles and dyons. The design may appear complicated but is essentially just a realistic embodiment of the concept illustrated in Figure 1C. The process is shown step-by-step, from two orthogonal viewing directions (a side and the top view). The central piece is a drive "wire" of underdoped $\mathrm{La}_{2-x} \mathrm{Sr}_{x} \mathrm{CuO}_{4}$ (UD-LSCO), a material that we conjecture to host dyons as low-energy excitations. It is currentbiased across the gold contacts $\mathbf{I}^{+}$and $\mathbf{I}^{-}$, shown in Figure 3 panel 12. A dc current in the range of micro-to milli-Amperes can be easily achieved. Take, for example, a UD-LSCO film with a representative resistivity $\rho=10 \mathrm{~m} \Omega \cdot \mathrm{cm}$, patterned into a bar that is $100 \mu \mathrm{m}$ long, $40 \mu \mathrm{m}$ wide and $25 \mathrm{~nm}$ thick; that would give the resistance $R=$ $10 \mathrm{k} \Omega$, and the current of $I=1 \mathrm{~mA}$ for a voltage bias $V=10 \mathrm{~V}$. These are quite comfortable values to work with using our standard experimental setups for transport measurements.

The second key part of our dyon-detector device is the pick-up ring or coil used to detect the circumferential electric field, or equivalently, the induced voltage at the end points of a ring that is not completely closed. Allowing for the possibility that this circumferential field may be small, we may therefore wish to use a low frequency $(1-100 \mathrm{KHz})$ ac drive current and lock-in detection techniques. As the preferred material for the pick-up coil, we choose optimally doped (OP) LSCO. Apart from a low dissipation, OPLSCO has the advantage of excellent crystallographic and chemical compatibility with the material (UD-LSCO) we use for the central drive wire. Since the drive wire must be electrically isolated from the pick-up coil, for this we can use thin layers of $\mathrm{LaSrAlO}_{4}$ (LSAO), the same material that we use for the substrate. We have already demonstrated the capability to grow LSCO epitaxially on LSAO 
and vice versa, with excellent crystal quality and atomically abrupt interfaces. Moreover, ultrathin (1-2 UC) LSAO layers are insulating enough to completely disrupt out-of-plane supercurrent and decouple LSCO layers.

The experiment described above has been designed using LSCO as the compound of choice. Note that fabrication of heterostructures described in Figure 2 and Figure 3 requires three materials with quite disparate properties-the active underdoped cuprate "wire" that hosts dyons, the HTS superconducting layers used to make the detection coil, and the insulating spacer layers that isolate the wire from the coil. One constraint on the choice of these three materials is that they need to be perfectly compatible in terms of their crystallographic structure, chemical composition, and electronic properties, so that the heterostructure can be grown with the high quality of each layer and with atomically sharp interfaces. The main advantage of LSCO is that it can be used for all three constituent materials, just by adjusting the Sr doping level-underdoped LSCO for the active wire, optimally doped LSCO for the HTS coil, and undoped LCO (without any $\mathrm{Sr}$ ) as the insulating spacer. Technically, this is called quasi-homo-epitaxy, and it guarantees essentially perfect compatibility of layers with quite disparate transport properties.

If the proposed experiment provides a positive outcome, it would be questioned whether this phenomenon is relevant for understanding of HTS in cuprates. If it is, then indeed it should be generic to all HTS cuprates, and one would wish to replicate the same experiment using all other key families of HTS cuprates, including $\mathrm{YBa}_{2} \mathrm{Cu}_{3} \mathrm{O}_{7}$ (YBCO), $\mathrm{Bi}_{2} \mathrm{Sr}_{2} \mathrm{Ca}_{n} \mathrm{Cu}_{\mathrm{n}+1} \mathrm{O}_{2 \mathrm{n}+2}$ (BSCCO), $\mathrm{Tl}_{\mathrm{m}} \mathrm{Ba}_{2} \mathrm{Ca}_{\mathrm{n}-1} \mathrm{Cu}_{\mathrm{n}} \mathrm{O}_{2 \mathrm{n}+\mathrm{m}+2}$ (TBCCO) and $\mathrm{Hg}_{2} \mathrm{Ba}_{2} \mathrm{Ca}_{\mathrm{n}} \mathrm{Cu}_{\mathrm{n}+1} \mathrm{O}_{2 \mathrm{n}+2}$ (HBCCO) for the active "wire." Of these, we have already amply demonstrated that high-quality HTS films of YBCO and BSCCO (with $n=1,2,8$ ) can be grown by ALL-MBE. However, the choice of the adequate insulator material has yet to be determined, by some focused material-science experiments. As for Tl- and Hgbased cuprates, the situation is less favorable for ALL-MBE, since these compounds contain highly volatile constituent atoms (Tl and $\mathrm{Hg}$ ) that would evaporate out of the film once it is brought to the typical growth temperature $\left(600-750^{\circ} \mathrm{C}\right)$ needed to achieve good crystallinity. Hence, for these cuprate families, we would need a different experimental strategy and device design; at the moment, these are still under development, but appear feasible.

In the design shown in Figure 2, Figure 3 the top and bottom halves of the superconducting OP-LSCO coil are deliberately staggered, for clarity. Note that this is not necessary; once the reader understands the above device pattern, he/she can easily imagine the two halves being placed right on top of one another, while keeping just the voltage gold contacts $\mathbf{V}^{+}$and $\mathbf{V}^{-}$staggered. Other variations of this design, including but not limited to drive wires and pickup coils of different dimensions, are also easily conceived. We have demonstrated the capability to fabricate LSCO nanowires down to $100 \mathrm{~nm}$ in width and $1 \mathrm{~nm}$ in the thickness of the HTS layer. On the other hand, our design features large-area gold contact, to ensure low contacts resistances, several orders of magnitude smaller than the drive wire resistance.

The key remaining step is to estimate the (order-ofmagnitude of) expected signal, i.e., the voltage $V$ between

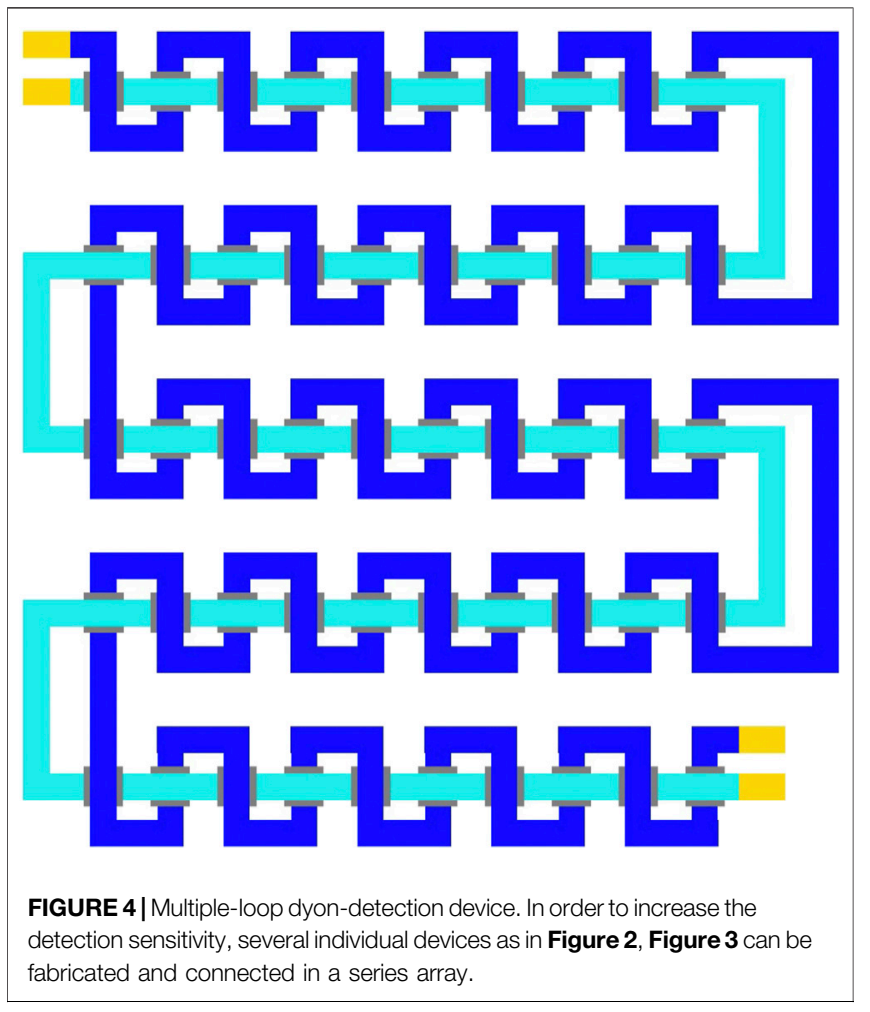

the contacts $\mathbf{V}^{+}$and $\mathbf{V}^{-}$. We can choose $I=1 \mathrm{~mA}$ as a representative drive current, and the device geometry and dimensions as specified in Figure 2, Figure 3. We could then evaluate $V$ from Eq. (18), if we only knew the exact form of the function $f$; however, this is not known precisely at the moment. Nevertheless, we know that $\pi / f^{2}$ is a monotonic function of the (relative) doping level $p / p_{\max }$, and that $f=1$ in OP-LSCO and $f \gg 1$ in extreme UD-LSCO. Assuming, e.g., that $f \sim 10,000$, from (Eq. 18) we get $V \sim 600 \mathrm{mV}$, which would be four to five orders of magnitude above our noise threshold $(\sim 10 \mathrm{nV})$. Hence, the signal/noise level should be excellent even if $f$ were a few more orders of magnitude larger.

For clarity, the device depicted in Figure 2, Figure 3 contains the simplest coil with just a single turn. It is straightforward, though, to generalize this design to a device that includes a multiple-turn coil, as illustrated in Figure 4. Note that compared with the single-turn device this design in fact requires no additional processing steps, just a little more complex lithographic mask. The main advantage here is that in this way one could increase further the signal, basically multiplying it by the number of turns, which can easily be made of the order of 10-100.

\section{SUMMARY AND OUTLOOK}

We have presented a theoretical conjecture that low-energy excitation in UD-LSCO should behave as dyons, carrying both the electric and magnetic charges, and outlined a detailed description of a new experimental device specifically 
designed to enable detection of such excitations. By virtue of the advanced synthesis and lithographic techniques that have been developed in the last few decades, this experiment has now become doable. It should be a real "smoking-gun" yes-orno experiment. A positive result promises to uniquely prove the conjecture and the existence of magnetic monopoles in UD-LSCO, which would be an exciting and important discovery of a new state of condensed matter. We hope that our work will motivate and stimulate further theoretical and experimental efforts in this direction.

\section{DATA AVAILABILITY STATEMENT}

The original contributions presented in the study are included in the article/Supplementary Material, further inquiries can be directed to the corresponding authors.

\section{REFERENCES}

1. Goddard P, Olive DI. Magnetic Monopoles in Gauge Field Theories. Rep Prog Phys (1978) 41:1357-437. doi:10.1088/0034-4885/41/9/001

2. Polyakov AM. Compact Gauge fields and the Infrared Catastrophe. Phys Lett B (1975) 59:82-4. doi:10.1016/0370-2693(75)90162-8

3. Diamantini MC, Trugenberger CA, Vinokur VM. Quantum Magnetic Monopole Condensate. Commun Phys (2021) 4:25. doi:10.1038/s42005-02100531-5

4. Tinkham M. Introduction to Superconductivity. Mineola (N.Y.): Dover Publications (1996).

5. Diamantini MC, Trugenberger CA, Vinokur VM. Confinement and Asymptotic freedom with cooper Pairs. Commun Phys (2018) 1:77. doi:10.1038/s42005-018-0073-9

6. Timusk T, Statt B. The Pseudogap in High-Temperature Superconductors: an Experimental Survey. Rep Prog Phys (1999) 62:61-122. doi:10.1088/00344885/62/1/002

7. Damascelli A, Hussain Z, Shen Z-X. Angle-resolved Photoemission Studies of the Cuprate Superconductors. Rev Mod Phys (2003) 75:473-541. doi:10.1103/ revmodphys.75.473

8. Norman MR, Pines D, Kallin C. The Pseudogap: Friend or Foe of highTc? Adv Phys (2005) 54:715-33. doi:10.1080/00018730500459906

9. Deutscher G. Andreev-Saint-James Reflections: A Probe of Cuprate Superconductors. Rev Mod Phys (2005) 77:109-35. doi:10.1103/ revmodphys.77.109

10. Chen Q, Stajic J, Tan S, Levin K. BCS-BEC Crossover: From High Temperature Superconductors to Ultracold Superfluids. Phys Rep (2005) 412:1-88. doi:10.1016/j.physrep.2005.02.005

11. Lee PA, Nagaosa N, Wen X-G. Doping a Mott Insulator: Physics of HighTemperature Superconductivity. Rev Mod Phys (2006) 78:17-85. doi:10.1103/ revmodphys.78.17

12. Hüefner S, Hossain MA, Damascelli A, Sawatzky GA Two Gaps Make a HighTemperature Superconductor? Rept Prog Phys (2008) 71:062501.

13. Keimer B, Kivelson SA, Norman MR, Uchida S, Zaanen J. From Quantum Matter to High-Temperature Superconductivity in Copper Oxides. Nature (2015) 518:179-86. doi:10.1038/nature14165

14. Vishik IM. Photoemission Perspective on Pseudogap, Superconducting Fluctuations, and Charge Order in Cuprates: a Review of Recent Progress. Rep Prog Phys (2018) 81:062501. doi:10.1088/1361-6633/aaba96

15. Robinson NJ, Johnson PD, Rice TM, Tsvelik AM. Anomalies in the Pseudogap Phase of the Cuprates: Competing Ground States and the Role of Umklapp Scattering. Rep Prog Phys (2019) 82:126501. doi:10.1088/1361-6633/ab31ed

16. Diamantini MC, Trugenberger CA, Vinokur VM. Topological Nature of High Temperature Superconductivity. Adv Quan Tech (2021) 4:2000135. doi:10.1002/qute.202000135

\section{AUTHOR CONTRIBUTIONS}

$\mathrm{IB}, \mathrm{MD}, \mathrm{CT}$, and VV conceived the work, MD, CT, and VV performed calculations. IB and $\mathrm{AB}$ designed the experiment, all authors wrote the manuscript.

\section{FUNDING}

The authors declare that this study received funding from Terra Quantum AG. The funder was not involved in the study design, collection, analysis, interpretation of data, the writing of this article, or the decision to submit it for publication. $\mathrm{AB}$ was supported by the Gordon and Betty Moore Foundation's EPiQS Initiative through Grant GBMF9074. IB was supported by the United States Department of Energy, Basic Energy Sciences, Materials Sciences and Engineering Division.

17. Diamantini MC, Sodano P, Trugenberger CA. Gauge Theories of Josephson junction Arrays. Nucl Phys B (1996) 474:641-77. doi:10.1016/0550-3213(96) 00309-4

18. Vinokur VM, Baturina TI, Fistul MV, Mironov AY, Baklanov MR, Strunk C. Superinsulator and Quantum Synchronization. Nature (2008) 452:613-5. doi:10.1038/nature06837

19. Diamantini MC, Postolova SV, Mironov AY, Gammaitoni L, Strunk C, Trugenberger CA, et al. Direct Probe of the interior of an Electric Pion in a cooper Pair Superinsulator. Commun Phys (2020) 3:142. doi:10.1038/s42005020-00410-5

20. Treiman SB, Jackiw R, Zumino B, Witten E. Current Algebra and Anomalies. Singapore: World Scientifc (1985).

21. Wilczek F. Two Applications of Axion Electrodynamics. Phys Rev Lett (1987) 58:1799-802. doi:10.1103/physrevlett.58.1799

22. Sankar R. Topological Insulators: A Review. arXiv:1804.06471 (2018).

23. Vishwanath A, Senthil T. Physics of Three-Dimensional Bosonic Topological Insulators: Surface-Deconfined Criticality and Quantized Magnetoelectric Effect. Phys Rev X (2013) 3:011016. doi:10.1103/physrevx.3.011016

24. Deser S, Jackiw R, Templeton S. Three-dimensional Massive Gauge Theories. Phys Rev Lett (1982) 48:975-8. doi:10.1103/physrevlett.48.975

25. Witten E. Dyons of Charge E $9 / 2 \pi$. Phys Lett B (1979) 86:283-7. doi:10.1016/ 0370-2693(79)90838-4

26. Chen X, Gu Z-C, Liu Z-X, Wen X-G. Symmetry Protected Topological Orders and the Group Cohomology of Their Symmetry Group. Phys Rev B (2013) 87: 155114. doi:10.1103/physrevb.87.155114

27. Lu Y-M, Vishwanath A. Theory and Classification of Interacting Integer Topological Phases in Two Dimensions: a Chern-Simons Approach. Phys Rev B (2012) 86:125119. doi:10.1103/physrevb.86.125119

28. Chalker JT, Coddington PD. Percolation, Quantum Tunnelling and the Integer Hall Effect. J Phys C: Solid State Phys (1988) 21:2665-79. doi:10.1088/00223719/21/14/008

29. Barisic N, Chan MK, Li Y, Yu G, Zhao X, Dressel M, et al. Universal Sheet Resistance and Revised Phase Diagram of the Cuprate High-Temperature Superconductors. Proc Natl Acad Sci (2013) 110:12235-40. doi:10.1073/ pnas. 1301989110

30. Proust C, Vignolle B, Levallois J, Adachi S, Hussey NE. Fermi Liquid Behavior of the In-Plane Resistivity in the Pseudogap State of YBa2Cu4O8. Proc Natl Acad Sci USA (2016) 113:13654-9. doi:10.1073/ pnas. 1602709113

31. Sato Y, Kasahara S, Murayama H, Kasahara Y, Moon E-G, Nishizaki T, et al. Thermodynamic Evidence for a Nematic Phase Transition at the Onset of the Pseudogap in YBa2Cu3Oy. Nat Phys (2017) 13:1074-8. doi:10.1038/nphys4205

32. Kivelson SA, Fradkin E, Emery VJ. Electronic Liquid-crystal Phases of a Doped mott Insulator. Nature (1998) 393:550-3. doi:10.1038/31177

33. Xia J, Schemm E, Deutscher G, Kivelson SA, Bonn DA, Hardy WN, et al. Polar KerrEffect Measurements of the High-TemperatureYBa2Cu3O6+xSuperconductor: 
Evidence for Broken Symmetry Near the Pseudogap Temperature. Phys Rev Lett (2008) 100:127002. doi:10.1103/PhysRevLett.100.127002

34. Logvenov G, Gozar A, Bozovic I. High-temperature Superconductivity in a Single Copper-Oxygen Plane. Science (2009) 326:699-702. doi:10.1126/ science. 1178863

35. Zhou H, Yacoby Y, Butko VY, Logvenov G, Bozovic I, Pindak R. Anomalous Expansion of the Copper-Apical-Oxygen Distance in Superconducting Cuprate Bilayers. Proc Natl Acad Sci (2010) 107:8103-7. doi:10.1073/pnas.0914702107

36. Zhou P, Chen L, Liu Y, Sochnikov I, Bollinger AT, Han M-G, et al. Electron Pairing in the Pseudogap State Revealed by Shot Noise in Copper Oxide Junctions. Nature (2019) 572:493-6. doi:10.1038/s41586-0191486-7

37. Litombe NE, Bollinger AT, Hoffman JE, Božović I. La 2 - X Sr X CuO 4 Superconductor Nanowire Devices. Physica C: Superconductivity its Appl (2014) 506:169-73. doi:10.1016/j.physc.2014.06.010

Conflict of Interest: VV was employed by Terra Quantum AG, CT was employed by SwissScientific Technologies
The remaining authors declare that the research was conducted in the absence of any commercial or financial relationships that could be construed as a potential conflict of interest.

Publisher's Note: All claims expressed in this article are solely those of the authors and do not necessarily represent those of their affiliated organizations, or those of the publisher, the editors, and the reviewers. Any product that may be evaluated in this article, or claim that may be made by its manufacturer, is not guaranteed or endorsed by the publisher.

Copyright $\odot 2022$ Diamantini, Trugenberger, Bollinger, Vinokur and Božović. This is an open-access article distributed under the terms of the Creative Commons Attribution License (CC BY). The use, distribution or reproduction in other forums is permitted, provided the original author(s) and the copyright owner(s) are credited and that the original publication in this journal is cited, in accordance with accepted academic practice. No use, distribution or reproduction is permitted which does not comply with these terms. 